\title{
Time-dependent pulsing of microfluidic pumps to enhance 3D bioprinting of peptide bioinks
}

Khan, Zainab, Kahin, Kowther, Hauser, Charlotte A.

Zainab Khan, Kowther Kahin, Charlotte A. E. Hauser, "Time-dependent pulsing of microfluidic pumps to enhance 3D bioprinting of peptide bioinks," Proc. SPIE 11637, Microfluidics, BioMEMS, and Medical Microsystems XIX, 1163709 (5 March 2021); doi: 10.1117/12.2578830

SPIE. Event: SPIE BiOS, 2021, Online Only 


\title{
Time-Dependent Pulsing of Microfluidic Pumps to Enhance 3D Bioprinting of Peptide Bioinks
}

\author{
Zainab Khan', Kowther Kahin', and Charlotte A. E. Hauser ${ }^{*}$ \\ ${ }_{1}$ Department of Biological and Environmental Science and Engineering, King Abdullah University \\ for Science and Technology, Thuwal, 23955- 6900, Saudi Arabia \\ *Corresponding author: Charlotte A. E. Hauser (charlotte.hauser@kaust.edu.sa)
}

\begin{abstract}
Using bioinks for 3D bioprinting of cellular constructs remains a challenge due to factors including viscosity, fluid dynamics and shear stress. The encapsulation of cells within the bioinks directly affects the quality of 3D bioprinting and microfluidic pumping is a commonly used supporting approach. The accuracy of microfluidic pumps can be further improved by introducing various mixing techniques. However, many of these techniques introduce complex geometries or external fields. In this study, we used a simple control technique of time-dependent pulsing for instant gelation of the peptide bioinks and observed its effect during the bioprinting process. Various time-dependent periodic signals are imposed on to a stable flow cycle and the effects are analyzed. The microfluidic pumps are programmed with different flow patterns represented by low frequency sinusoidal pulses, ramp inputs, and duty cycle pulses. Different combinations of these pulses are tested to achieve an optimal pulse for improved quality of printed constructs. Time-varied pulsing of microfluidic pumps, particularly as square waveforms, is found to provide better continuous flow and avoid material buildup within the extruder unit when compared to pumping at a constant flow rate with manual tuning. Clogging is avoided since the gelation rate is periodically reduced which avoids gel clumps in the printed constructs. This study substantially improves the use of suitable peptide bioinks, standardizes the 3D bioprinting process, and reduces clogging and clumping during printing. Our findings allow for printing of more accurate and complex constructs for applications in tissue engineering, such as skin grafting, and other regenerative medical applications.
\end{abstract}

Keywords: 3D Bioprinting, Bioink, Hydrogel, Ultrashort Peptides, Syringe pumps, Tissue Fabrication

\section{INTRODUCTION}

Microfluidic studies have made considerable progress in the past two decades. They enhanced capabilities in handling complex fluidics when compared to any macroscopic system. In spite of sharing common grounds with macroscopic systems in terms of physics and chemistry principles, some of the effects are insignificant on small scales and some are dominant in microfluidics. Any engineer working on devices for processing liquids learns to develop intuition that the physics occurring in such devices should be neglected when shifting to small-scale systems ${ }^{[1]}$.Practical examples of microfluidic applications are flow of chemistry and drug discovery. Manufacturing techniques, flow control and mixing are some of the more challenging engineering applications in microfluidics. ${ }^{[2]}$

Having channels to transport fluids through microfluidic syringe pumps devices can easily provide several functions. However, these devices face numerous difficulties starting with clogging, leakages and formation of undesired bubbles. Incorporating mechanisms to eliminate bubbles and to have pulse-free fluidic delivery is a key element in the development of dependable microfluidic devices ${ }^{[3]}$. One of the most used syringe pump systems that tackled the mentioned issues is the Harvard Apparatus; it has been used by several researchers in the past 20 years. More recently, the development of an 
open-source syringe pump system has surged, with most of these systems using GUI-based interfaces. Cellix ${ }^{\circledR}$ is one such company that provided solutions aiding researchers' experimental set-ups by incorporating user-friendly software ${ }^{[4]}$.

Regulating flow rate is essential for most microfluidic devices. Syringe pumps, peristaltic pumps and pressure-driven pumps are the commonly used systems as they convey flow conveniently and offer excellent control of fluids at the microfluidic scale. Peristaltic pumps distribute a pulsatile flow ${ }^{[5]}$. They contain a very elastic tubing which is looped at the pump's rotor; flow rates range from $2 \mu \mathrm{L} / \mathrm{min}-10 \mu \mathrm{L} / \mathrm{min}$. On the other hand, syringe pumps are generally used in biomedical fields due to their lower range of flow rates, which can reach as low as $0.012 \mathrm{~nL} / \mathrm{min}$. Pressure regulating pumps control flow rates by applying pressure in a sealed tank. The flow rate can be measured by adding a flowmeter in the fluidic line which provides feedback to adjust the pressure ${ }^{[5]}$. Time-varied pulsing of microfluidic pumps is found to provide better continuous flow and avoid material buildup within the extruder unit when compared to pumping a constant flow from the pumps. Clogging is avoided since the gelation rate is periodically reduced which avoids gel clumps in the printed constructs.

Bioinks based on ultrashort peptides composed of 3-7 natural amino acids boast great potential due to their natural but synthetic properties and their salt-enhanced instantaneous gelation ${ }^{[6-10]}$. Unlike polymers or gelatin and alginate-based bioinks, these peptide bioinks eliminate harmful processes of UV light and chemical exposures for post-printed crosslinking of the bioinks. These features, with the advantage of being synthetic but body-like, biocompatible and offering facile biochemical synthesis, make them promising candidates for 3D bioprinted skin grafts and regenerative tissues. However, 3D bioprinting of peptide bioinks proves to be a challenge due to its low viscosity and rapid gelation. While most commercial 3D bioprinters depend on pneumatic extrusion, cartridge extrusion units with pre-mixed bioinks, and temperature-dependent protocols for solidification of bioinks, these protocols do not allow the direct encapsulation of cells within the peptide hydrogel. Peptide bioink formation requires an instantaneous mixing and printing approach in the presence of the cells that are selected for printing, due to its rapid gelation. Hence, a custom 3D bioprinting system was developed with a 3D bioprinting robotic arm, three microfluidic pumps for peptide bioink formation, and a customdesigned nozzle with a controlled mixing region for bioink extrusion (Fig. 1a). ${ }^{[11] .}$

In order to standardize the 3D bioprinting process and automate the extrusion mechanism to eliminate clogging, this study introduced time-dependent pulsing into the microfluidic pumping process, without the use of cells in order to focus on the mechanical properties of the bioink. This allows the pumps to mix the bioink components and gradually adjust flow rates at specific intervals for extrusion in order to form consistent gel and avoid clumping and clogging. Different waveforms were tested including sinusoidal waveforms, ramp inputs, and square waveforms. The results for each waveform were compared with those achieved with manually tuned and constant flow rates. The results of this study aim to improve the mechanical properties and printing resolution of 3D bioprinted constructs with peptide bioinks as well as optimize the repeatability of the robotic 3D bioprinter.

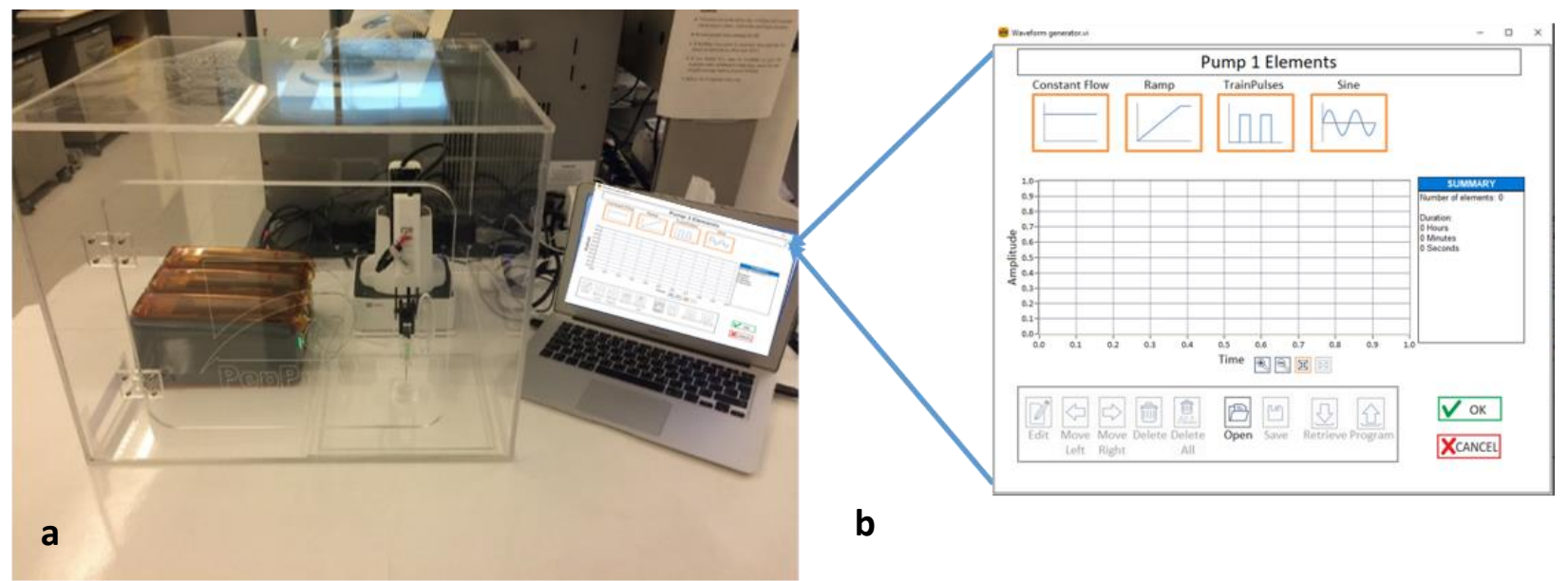

Figure 1 (a) 3D Bioprinting system with microfluidic pumps and custom dual coaxial nozzle (b) Close-up image of the Smartflow software panel 


\section{METHODOLOGY}

The aim of this research was to improve the quality of the 3D printed constructs by introducing automated time-dependent pulsing of microfluidic pumps, therefore optimizing repeatability of the robotic 3D bioprinter and reducing intervention by relying on automation. Five comparative experiments were conducted using SmartFlo Software (Fig. 1b). Different waveforms were tested including sinusoidal waves, ramp inputs, and square waves. The results for each waveform were compared with those achieved with constant and manually tuned flow rates. All experiments were independently repeated three times. Cells were not introduced in the printing process to conserve biological material during optimization of the mechanical printability.

Prior to starting the experiments, several components of the 3D bioprinting system were set up. Firstly, the robotic 3D bioprinter was set in 3D printing mode (Fig. 1a). Secondly, a custom dual coaxial nozzle was fabricated. Thirdly, the test structure was created using G-code Fig. 2. The test file was designed as a series of connected parallel lines to be able to observe the timepoints of clogging and clumping during printing. Cellix ExiGo® pumps were fitted with the necessary tubings to attach to the nozzle. These pumps were chosen as the Labview-based GUI interface allows pumping with a combination of various flow profiles and automation by programming pumps in advance.

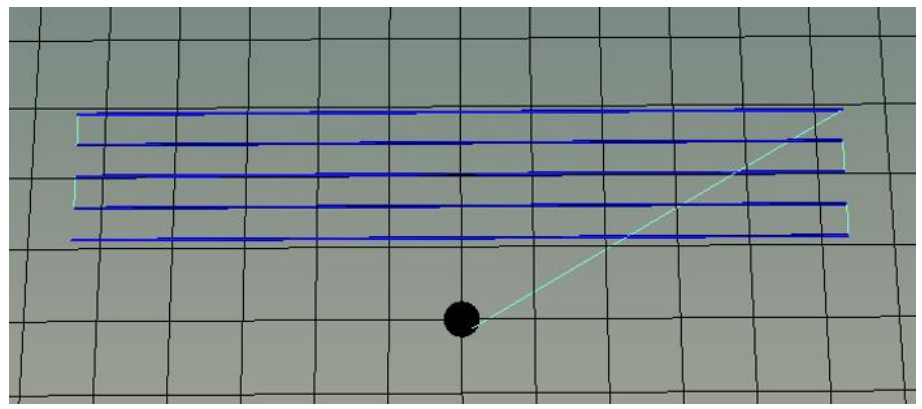

Figure $2 \mathrm{G}$-code preview of single layer test construct, 5 parallel lines of length $10 \mathrm{~cm}$ to observe bioink thread continuity

For each printing experiment, bioink was prepared using $15 \mathrm{mg}$ of IVZK peptide mixed in $1 \mathrm{ml}$ of water and sonicated. Finally, two Cellix ${ }^{\circledR}$ syringe pumps were loaded with the peptide mixture and 5x PBS solution as a buffer to activate the gelation. The optimal flow rate range of both syringe pumps had been determined in previous studies to ensure that the liquid solutions emerge at a reasonable speed in coordination with the printing speed ${ }^{[12]}$. The optimized flow rates ranged from $55 \pm 5 \mu \mathrm{l} / \mathrm{min}$ for the peptide hydrogel and $20 \pm 5 \mu \mathrm{l} / \mathrm{min}$ for phosphate-buffered saline solution (PBS).

The auto-tuning trials included the following waveforms: constant flow with and without manual tuning (as a control reference), sinusoidal waveforms, ramp inputs, and square waveforms. A set of flow profiles was created consisting of different combinations of each waveform in the SmartFlo® software. Each flow profile was tested within the previously determined optimal ranges of pumping for bioink formation and multiple samples were printed. Based on the results, the flow rate ranges, duty cycles, and periods were adjusted for further testing. For each experiment, the following parameters were assessed during printing - continuity of gel, extruded volume, strain on nozzle, and interruptions due to clogs and clumps.

\subsection{Constant Flow Profile}

As a control reference point, the first flow profiles were set to the original printing parameters used in our 3D bioprinting process. This consisted of constant flow at a set flow rate with manual tuning within a range of $\pm 5 \mu 1 / \mathrm{min}$. To observe the 
effect of flow rate adjustment while printing, samples were printed at constant flow with and without manual tuning. The set flow rates were $55 \mu \mathrm{l} / \mathrm{min}$ and $15 \mu \mathrm{l} / \mathrm{min}$, respectively. With manual tuning, the flow rates were adjusted after approximately 1.5 minute of pumping. The flow rates fluctuated between 50 and $55 \mu \mathrm{l} / \mathrm{min}$ for the peptide and $15-20$ $\mu \mathrm{l} / \mathrm{min}$ for the buffer. The manually tuned structure was compared with the 3 automated waveforms in terms of structure fidelity and printing resolution.

\subsection{Ramp Inputs}

The first automated waveform to be tested was set with ramp inputs at specific time intervals. These inputs allow gradual increase of flow rate from a minimum to a maximum over a specific period of time. The flow profiles were created as a combination of constant flow with ascending and descending ramp inputs. The flow rate range was set at $45-55 \mu 1 / \mathrm{min}$ for peptide and 15-25 $\mu \mathrm{l} / \mathrm{min}$ for the PBS. Different time intervals for the ramp inputs were set at $25 \%, 50 \%$ and $75 \%$ of the total printing time. The total printing time was extracted from the 3D printing software and the time interval for each portion of the flow profile was calculated accordingly. Each test construct was observed and adjustments were made accordingly.

\subsection{Sinusoidal Waveform}

The sinusoidal waveform was tested with flow profiles of different amplitudes including 5, 10 and $15 \mu 1$. The offset was set accordingly to obtain flow rate ranges between $45-60 \mu \mathrm{l} / \mathrm{min}$ for peptide and $15-25 \mu \mathrm{l} / \mathrm{min}$ for pbs. Different period lengths were tested from 30 seconds to 2 minutes. The phase shift was kept at zero.

\subsection{Square Waveform}

The final automated waveform tested was the square waveform. Flow profiles were created with 5 and $10 \mu \mathrm{l} / \mathrm{min}$ increments and duty cycles of $25 \%, 50 \%$ and $75 \%$. Combined flow profiles were also tested where the peptide flow rate was kept constant while the PBS flow rate was set to a square waveform over a range of $15-20 \mu \mathrm{l} / \mathrm{min}$. In addition to the parallel line test file, a $2 \mathrm{~cm}$ grid shape was also printed for longer observation. The grid constructs were observed for $7 \min 40$ s with a $75 \%$ duty cycle.

\section{THE RESULTS}

The following section discusses the results obtained from comparative flow profile experiments. Table 1 shows the parameters used for each experiment presented in Fig. 3.

\subsection{Constant Flow Profile}

In the first experiment, the test file was printed according to the standard bioprinting protocol with manually tuned pumping at a constant flow rate. This print was used as a control reference point with the aim of obtaining better results with the automated time-dependent pumping. As can be seen in Figure 3a, microfluidic pumping at a constant flow rate without any adjustment increased blockage of the nozzle, therefore reduced the printing resolution. After about 1.5 min of pumping, the increased gelation would build up in the nozzle, resulting in clumps. If left unattended, these clumps would clog up the nozzle creating backflow and eventually leakage. According to our standard protocol, the solution to avoid this was by manually adjusting the flow rate of each pump by $\pm 5 \mu \mathrm{l} / \mathrm{min}$ as needed during printing to reduce the constant buildup of gel in the nozzle. This ensured continuous flow of gel and reduced the nozzle clogging as can be seen in (Fig. 3b). However, because the tuning was done manually, it was inaccurate and highly dependable on the intuition of the user to predict a clog beforehand and decide when to increase or decrease the flow rate of each pump. Hence, the automated waveforms were printed to be compared with manually tuned constant flow profiles in hopes of improving the printing reliability and resolution as well as eliminating inconsistent flow of gel and clogs in the nozzle. 


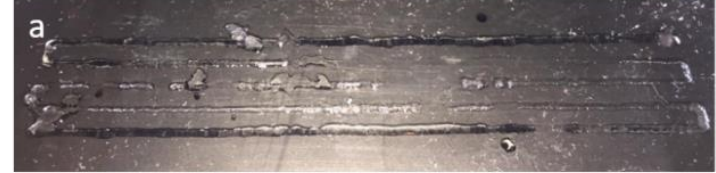

Constant Flow

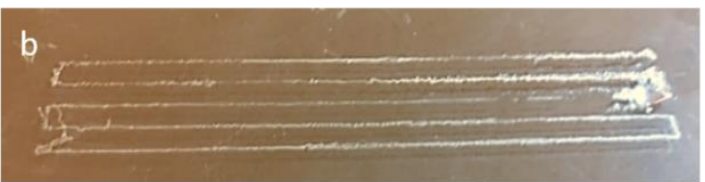

Constant Flow with Manual Tuning

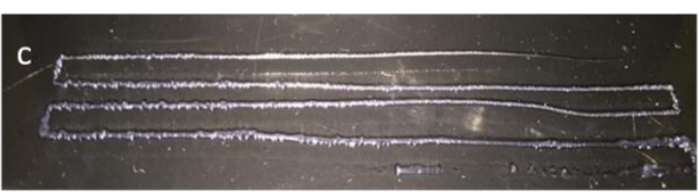

Ramp + Constant Flow

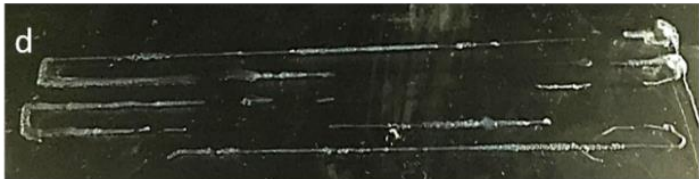

Sinusoidal Wave

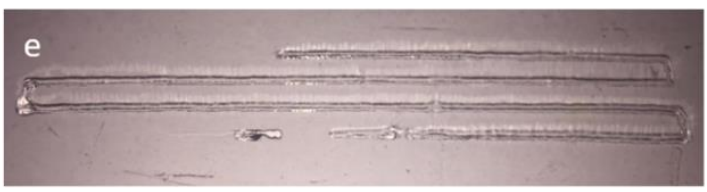

Square Wave
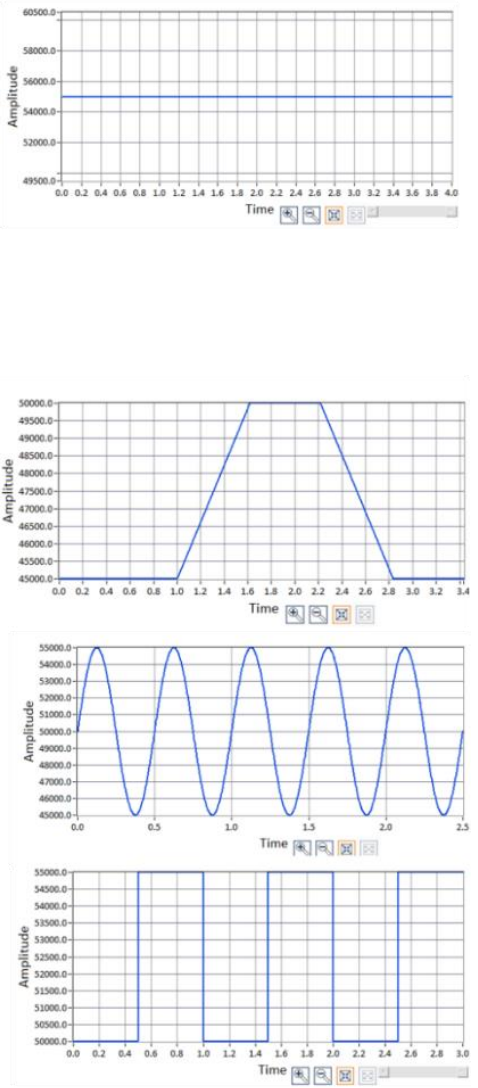

Figure 3 (a) 3D printed bioink strands at constant flow rate, $55 \mu 1 / \mathrm{min}$ peptide and $15 \mu \mathrm{l} / \mathrm{min}$ PBS (b) 3D printed bioink stands at constant flow rate with manual tuning, $50-55 \mu \mathrm{l} / \mathrm{min}$ Peptide and $15-20 \mu \mathrm{l} / \mathrm{min}$ PBS. Graph showing sample constant flow profile (right) (c) 3D printed bioink strands with flow profile of ramp inputs (50\% duty cycle) and constant flow (50\% duty cycle), Range of $45-55 \mu \mathrm{l} / \mathrm{min}$ Peptide, $15-25 \mu 1 / \mathrm{min}$ PBS, Graph showing sample ramp flow profile (right) (d) 3D printed bioink strands with sinusoidal pumping, $55-60 \mu 1 / \min$ Peptide, $15-20 \mu 1 / \min$ PBS, Period = 1 minute, Graph showing sample sinusoidal flow profile (right) (e) 3D printed bioink strands with square pulse pumping, $50-55 \mu 1 /$ min Peptide, $15-20 \mu \mathrm{l} / \mathrm{min}$ PBS, Graph showing sample square wave flow profile (right)

\subsection{Ramp Inputs}

Ramp inputs were introduced in the first automated waveform. After testing multiple flow profiles, it was observed that results improved when an increasing ramp input was introduced with a 50\% duty cycle over the period of total printing time. Clumps and clogs were considerably reduced. However, during the ramp intervals, the gradual increase in flow resulted in extrusion of flimsier gel which had trouble maintaining shape even as a straight line. Drifts were clearly noticeable in the printed constructs (Fig. 3c). Some samples also showed occasional gaps due to slowed gelation during intervals of flow rate adjustment. This would negatively affect printing resolution for larger constructs so other waveforms were explored. 
Table 1: Comparison of Manual and Automated Flow Profiles

\begin{tabular}{|c|c|c|c|c|c|}
\hline Trial & Shape & $\begin{array}{c}\text { Start Flowrate } \\
(\mu \mathrm{l} / \mathrm{min})\end{array}$ & $\begin{array}{l}\text { End Flowrate } \\
\qquad(\mu \mathrm{l} / \mathrm{min})\end{array}$ & Period (s) & Duty Cycle \\
\hline $\begin{array}{l}\text { (a) Constant } \\
\text { Flow }\end{array}$ & Lines & $\begin{array}{l}\text { Peptide - 55 } \\
\text { PBS - } 15\end{array}$ & $\begin{array}{l}\text { Peptide - 55 } \\
\text { PBS - } 15\end{array}$ & - & - \\
\hline $\begin{array}{l}\text { (b) Constant } \\
\text { Flow w/ Manual } \\
\text { Tuning }\end{array}$ & Lines & $\begin{array}{l}\text { Peptide - 50 } \\
\text { PBS - } 15\end{array}$ & $\begin{array}{l}\text { Peptide - 60 } \\
\text { PBS - } 25\end{array}$ & $40-45$ & Manual \\
\hline $\begin{array}{l}\text { (c) Ramp + } \\
\text { Constant }\end{array}$ & Lines & $\begin{array}{l}\text { Peptide - } 45 \\
\text { PBS - } 15\end{array}$ & $\begin{array}{l}\text { Peptide - } 55 \\
\text { PBS - } 25\end{array}$ & 142 & $50 \%$ \\
\hline (d) Sinusoidal & Lines & $\begin{array}{l}\text { Peptide - 55 } \\
\text { PBS - } 15\end{array}$ & $\begin{array}{l}\text { Peptide - 60 } \\
\text { PBS - 20 }\end{array}$ & 60 & $50 \%$ \\
\hline (e) Square Wave & Lines & $\begin{array}{l}\text { Peptide - 50 } \\
\text { PBS - } 15\end{array}$ & $\begin{array}{l}\text { Peptide - } 55 \\
\text { PBS - 20 }\end{array}$ & 60 & $50 \%$ \\
\hline
\end{tabular}

\subsection{Sinusoidal Waveform}

Flow profiles were created with sinusoidal waveforms and the test construct of four lines was printed. However, as observed with ramp inputs, the printed shape was uneven due to the gradual flow rate adjustment which slowed down gel formation. Although clumps were few, several gaps were noticed in the constructs despite adjusting amplitude, period and offsets. It was concluded that this was due to insufficient pumping at the minimum flow rates of the flow profile. The printing resolution and structure fidelity was found to be better in prints made with manually tuned pumping as compared to sinusoidal pumping (Fig. 3d).

Hence, multiple observations were made from the ramp and sinusoidal flow profile experiments. It was found that pulsed increments were more effective than gradual increments as pulses were a better replication of the manual tuning process. It was also concluded that the flow rate adjustments should only be in increments and decrements of $5 \mu 1 / \mathrm{min}$. Larger ranges negatively affected gelation and caused inconsistent extrusion of gel. Lastly, the initial minimum flow rate for some flow profiles was set to $45 \mu 1 / \mathrm{min}$ and this was found to be too low to obtain a sufficient amount of gel extrusion. These points were taken into consideration when testing the final mode, square waveform. 
Table 2: Parameter optimization of Square Wave Flow Profile

\begin{tabular}{|c|c|c|c|c|c|}
\hline Trial & Shape & $\begin{array}{l}\text { Start Flowrate } \\
(\mu \mathrm{l} / \mathrm{min})\end{array}$ & $\begin{array}{l}\text { End Flowrate } \\
\qquad(\mu \mathrm{l} / \mathrm{min})\end{array}$ & $\begin{array}{c}\text { Period } \\
\text { (s) }\end{array}$ & Duty Cycle \\
\hline Manual & Grid & $\begin{array}{l}\text { Peptide - } 55 \\
\text { PBS - } 15\end{array}$ & $\begin{array}{l}\text { Peptide }-50 \\
\text { PBS - } 25\end{array}$ & - & - \\
\hline $\begin{array}{l}\text { Square } \\
\text { Wave } 1\end{array}$ & Grid & $\begin{array}{l}\text { Peptide - } 50 \\
\text { PBS - } 15\end{array}$ & $\begin{array}{l}\text { Peptide }-55 \\
\text { PBS - } 20\end{array}$ & 115 & $50 \%$ \\
\hline $\begin{array}{l}\text { Square } \\
\text { Wave } 2\end{array}$ & Grid & $\begin{array}{l}\text { Peptide }-55 \\
\text { PBS - } 15\end{array}$ & $\begin{array}{l}\text { Peptide }-55 \\
\text { PBS - } 20\end{array}$ & 115 & $50 \%$ \\
\hline $\begin{array}{l}\text { Square } \\
\text { Wave } 3\end{array}$ & Grid & $\begin{array}{l}\text { Peptide }-55 \\
\text { PBS - } 15\end{array}$ & $\begin{array}{l}\text { Peptide }-50 \\
\text { PBS - } 20\end{array}$ & 115 & $50 \%$ \\
\hline $\begin{array}{l}\text { Square } \\
\text { Wave } 4\end{array}$ & Grid & $\begin{array}{l}\text { Peptide - } 55 \\
\text { PBS - } 15\end{array}$ & $\begin{array}{l}\text { Peptide - } 50 \\
\text { PBS - } 20\end{array}$ & 115 & $\begin{array}{c}\text { Peptide } 75 \% \\
\text { PBS 25\% }\end{array}$ \\
\hline
\end{tabular}

A

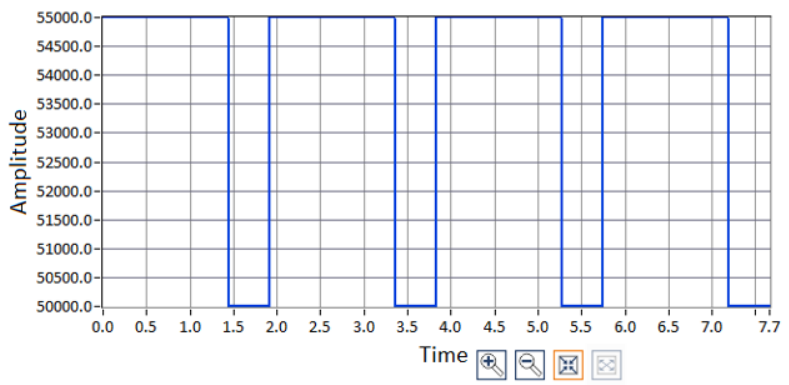

Pump 1 Peptide Hydrogel

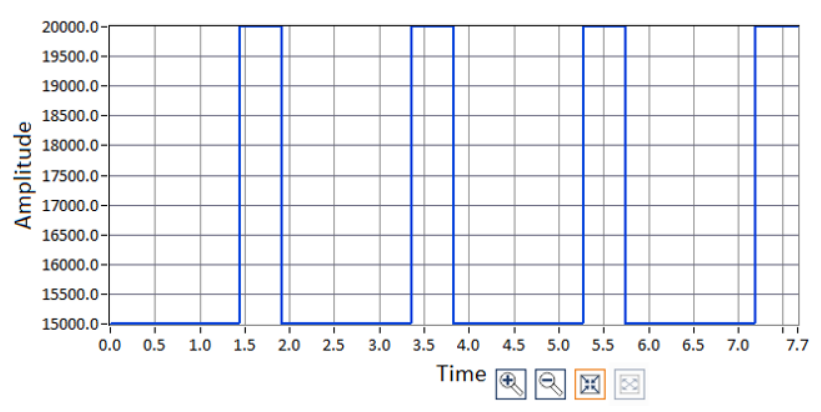

Pump 2 PBS

\section{B}

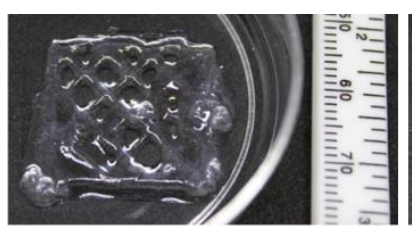

Constant + Manual Tuning

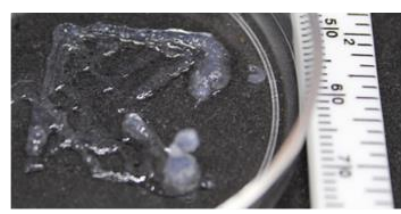

Square Wave 2

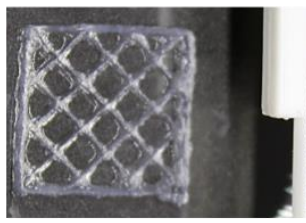

Square Wave 3

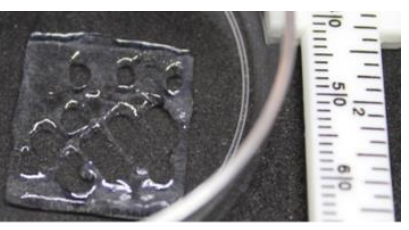

Square Wave 1
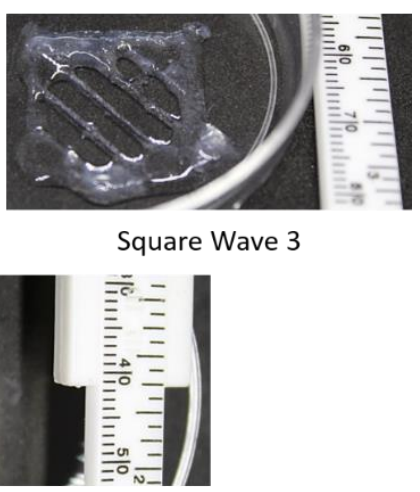

Square Wave 4 


\subsection{Square Waveform}

Similarly, flow profiles were set with square waveforms and the g-code testing file was printed. Promising results were obtained in the initial trials as no clogging or clumping was observed (Fig. 3e). Gaps were noticed at the beginning and end of the print but this was resolved by adjusting the parameters Table 2. After multiple trials, it was found that a key component for consistent gelation was to maintain a constant volume of $70 \mu \mathrm{l}$ throughout printing. Hence, alternating waveforms were set for both peptide and PBS pumps. For peptide, the duty cycle for the square waveform was set to $75 \%$ with a flow rate range of $50-55 \mu \mathrm{l} / \mathrm{min}$. For PBS, the duty cycle was set to $25 \%$ with a flow rate range of $15-20 \mu \mathrm{l} / \mathrm{min}$ (Fig. 4a). This improved the results dramatically as seen in the printed grid constructs (Fig. 4b). The optimized grid construct was then compared to a grid construct printed at a constant flow rate and manually tuned, which had clumps of gel scattered on the edges with excess PBS reducing resolution of the grid lines (Fig. 4b). The resolution of the printed construct optimized by square pulsing was visibly better and more defined. Fig 5 shows that the width of the bioink strands of the optimized auto-tuned construct is consistent throughout the print, which is a crucial factor for quality printing of larger constructs.
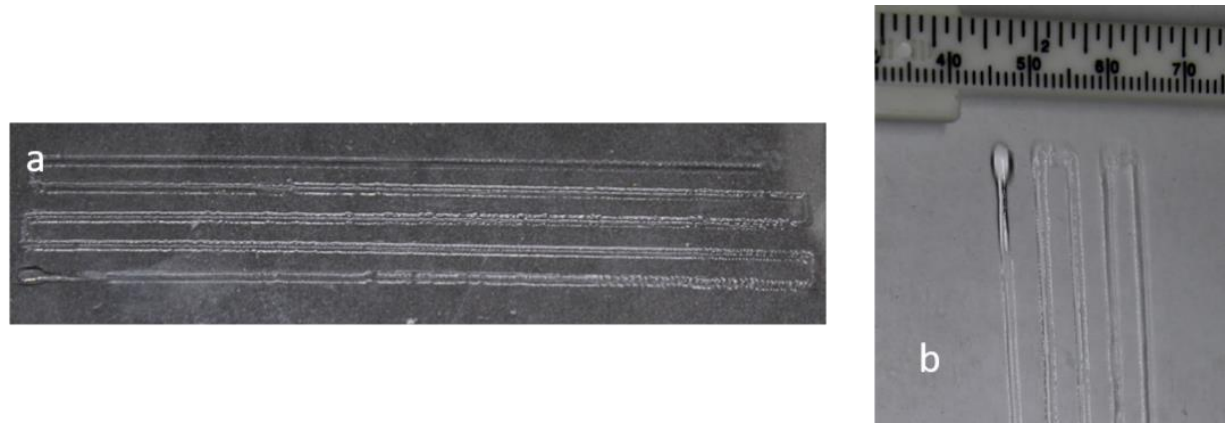

Figure 5 (a) 3D printed bioink strands with optimized square pulse pumping, $50-55 \mu \mathrm{l} / \mathrm{min}$ Peptide at $75 \%$ duty cycle, $15-20 \mu \mathrm{l} / \mathrm{min}$ PBS at $25 \%$ duty cycle, Period $=1: 55 \mathrm{~s}$ (b) Bioink strand width shows consistent flow throughout print

Hence, microfluidic pumping with time-dependent pulsing in square waveforms showed to improve printability and resolution when compared to manually tuned pumping. To confirm the results, several constructs were printed with the newly optimized settings. The outcome was found to be consistent with excellent resolution and finer defined lines.

\section{CONCLUSION}

This study substantially improves the use of selected peptide bioinks, automates the 3D bioprinting process, and reduces interruptions during printing. The goal to introduce automated time-dependent pulsing to achieve a consistent flow of bioink throughout the printing process was successfully achieved. Square pulses of alternating duty cycles for each pump were found to provide the best printing results with finer resolution, increased consistency, and reduced clumps, avoiding clogging and also guaranteeing a more homogenous distribution of the encapsulated cells within the bioink. These results demonstrate that automation of the pumping system allows printing of more accurate, complex and long-lasting constructs with improved mechanical properties. The findings of this study improve the printing process to generate cell-laden constructs for applications in tissue engineering, such as skin grafting, organoid formation and other regenerative medical applications. 


\section{ACKNOWLEDGEMENTS}

The research reported in this publication was supported by funding from the King Abdullah University of Science and Technology (KAUST). We would like to thank Hamed Albalawi for his support in designing the test files in SolidWorks.

\section{REFERENCES}

[1] Hardt, S., \& Schönfeld, F. [Microfluidic Technologies for Miniaturized Analysis Systems], Springer, New York, Chapter 1 (2007).

[2] Cheaib, F., Kekejian, G., Antoun, S., Cheikh, M., \& Lakkis, I., " Microfluidic mixing using pulsating flows," Microfluid. Nanofluidics, $20(5), 70$ (2016).

[3] Kang YJ, Yeom E, Seo E, Lee SJ.,” Bubble-free and pulse-free fluid delivery into microfluidic devices,”. Biomicrofluidics, 08 (1), 014102 (2014).

[4] "Microfluidic Solutions.” Wearecellix, 2 Feb. 1970, www.wearecellix.com/microfluidic-solutions.

[5] Peristaltic, Syringe and Pressure Microfluidic Pumps Comparison." Fluigent, 27 Jan. 2020, www.fluigent.com/resources/microfluidicexpertise/what-is-microfluidic/system-comparison-for-microfluidic-applications/.

[6] Susapto, H. H., Alhattab, D., Abdelrahman, S., Khan, Z., Alshehri, S., Kahin, K., Ge, R., Moretti, M., Emwas, A. H., \& Hauser, C. " Ultrashort Peptide Bioinks Support Automated Printing of Large-Scale Constructs Assuring Long-Term Survival of Printed Tissue Constructs," Nano Lett. (2021).

[7] Rauf S, Susapto HH, Kahin K, Alshehri S, Abdelrahman S, Lam JH, Asad S, Jadhav S, Sundaramurthi D, Gao X, Hauser CAE, " Self-assembling tetrameric peptides allow in situ 3D bioprinting under physiological conditions," J Mater Chem B. 9(4), 1069-1081 (2021).

[8] Wu, E. C., Zhang, S., \& Hauser, C. A., " Self-Assembling peptides As Cell-interactive Scaffolds," Adv. Funct. Mater 22(3), 456-468 (2011).

[9] Hauser, C. A., Deng, R., Mishra, A., Loo, Y., Khoe, U., Zhuang, F., Cheong, D. W., Accardo, A., Sullivan, M. B., Riekel, C., Ying, J. Y., \& Hauser, U. A. " Natural tri- to hexapeptides self-assemble in water to amyloid beta-type fiber aggregates by unexpected alpha-helical intermediate structures," PNAS 108(4), 1361-1366 (2011).

[10] Mishra, Y. Loo, R. Deng, Y.J. Chuah, H.T. Hee, J.Y. Ying, C.A. Hauser, " Ultrasmall natural peptides self-assemble to strong temperature-resistant helical fibers in scaffolds suitable for tissue engineering," Nano Today 6(3), 232-239, (2011).

[11] Kahin, K., Kahn, Z., Albagami, M., Usman, S., Bahnshal, S., \& Alwazani, H., Abdulmajid, M., Rauf, S., \& Hauser, C "Development of a robotic 3D bioprinting and microfluidic pumping system for tissue and organ engineering," Proc. SPIE 10875, 130 -- 139 (2019).

[12] Khan, Z., Kahin, K., Rauf, S., Ramirez-Calderon, G., Papagiannis, N., Abdulmajid, M., \& Hauser, C." Optimization of a 3D bioprinting process using ultrashort peptide bioinks," Int. J. Bioprinting, 5(1), 173 (2018). 